\title{
História da Esclerose Lateral Amiotrófica no Brasil
}

\author{
History of Amyotrophic Lateral Sclerosis in Brazil
}

\begin{abstract}
Abrahão Augusto Juviniano Quadros
Fisioterapeuta, Mestre em Ciências da Saúde pela Universidade Federal de São Paulo - Escola Paulista de Medicina. Presidente da ABRELA - Associação Brasileira de esclerose Lateral Amiotrófica
\end{abstract}

A esclerose lateral amiotrófica ainda é pouco conhecida no Brasil, mesmo após 97 anos da primeira descrição da doença em nosso país. Embora sendo considerada como uma doença rara a ELA não afeta somente a pessoa com a doença. Ela causa um grande impacto social e econômico na família e na comunidade.

Esta abordagem apresenta três momentos da a história da ELA no Brasil: 1) Os primeiros estudos - de 1909 até 1935, 2) Estudos epidemiológicos - de 1983 até 2002 e, 3) Atuação da Associação Brasileira de Esclerose Lateral Amiotrófica - ABRELA.

1 - Os Primeiros Estudos - 1909 a 1935

Este período é caracterizado por estudos de casos clínicos, que proporcionaram as primeiras contribuições para o entendimento da manifestação da ELA em nosso meio. Os estudos se concentraram nos Estados do Rio de Janeiro, Rio Grande do Sul e São Paulo, conforme mostrado na tabela 1 .

A primeira descrição da esclerose lateral amiotrófica no Brasil coube ao Dr. Cypriano de Souza Freitas, professor de anatomia e fisiologia patológica da Faculdade de Medicina do Rio de Janeiro. Conhecedor na época de quatro casos de ELA. Escolheu para apresentar no IV Congresso Médico Latino Americano na cidade do Rio de Janeiro em agosto 1909, observações e comentários sobre um caso de ELA e em novembro do mesmo ano publicou no Brazil-Médico com o título "Sobre um caso de esclerose lateral amyotrophica", um caso clínico definido por ele como assas interessante, pela:

1) Raridade da afecção entre nós;

2) Idade de início dos sintomas;

3) Dificuldade de formular, desde o começo, um diagnóstico preciso, assumindo os fenômenos de uma forma frustra, bem diversa da forma clássica. 
Tratava-se de paciente do sexo masculino, branco, 73 anos de idade, sem antecedentes hereditários dignos de referência. Trabalhava em especulações financeiras e praticava atividade física sendo definido como "bom andador".

Começou a sentir ligeiro enfraquecimento da mão direita, sobretudo dos dedos. A fraqueza evoluiu gradualmente acompanhada de atrofia dos músculos da mão.

Examinado quatro meses após o início dos sintomas, apresentava paresia bem acentuada do membro superior direito. Atrofia muitíssimo acentuada na região tenar e acentuada na região hipotenar e dos interósseos. Apresentava também atrofia na musculatura do antebraço, braço e cintura escapular, fasciculação nas regiões atrofiadas, leve espasticidade e movimentos espasmódicos nas mãos. A musculatura do membro superior direito, da face, pescoço, tronco, e membros inferiores estava íntegra e com força normal.

O reflexo estava vivo no membro afetado e normal nos outros segmentos. A sensibilidade estava normal em todo o corpo. Os sentidos intactos, inteligência perfeita, sem dificuldade para comer e digerir e função esfincteriana normal.

Reavaliado, três meses mais tarde, apresentava atrofia nos músculos da mão esquerda com presença de fasciculação, sensibilidade normal e reflexos ora se mostravam vivos tanto nos membros superiores como os patelares, mas sem sinal de Babinski.

Referiu que as vezes tinha embaraço na pronúncia das palavras. Ao exame apresentava os movimentos de lábios e língua perfeitos, véu do palato simétrico, reflexo faríngeo normal, porém o reflexo masseterino estava vivo.

Um mês mais tarde, sofreu um forte ataque de gripe e ficou acamado por muitos dias. Após a recuperação da gripe, apresentava agravamento dos sintomas da ELA, com aparecimento de atrofia nos músculos do tronco e paresia nos membros inferiores, reflexos patelares exaltados (maior à direta), continuando a ausência do sinal de Babinski. Apareceram também dificuldade para deglutir, fasciculação de língua, impossibilidade de assobiar, insuficiência respiratória e acessos de sufocação, sobretudo a noite.

O período de avaliação foi de oito meses, de janeiro a agosto de 1909. Após a primeira avaliação clínica, o Dr. Cypriano confirma o diagnóstico de ELA com o seguinte comentário: “A vista da amiotrofia e da insignificância dos fenômenos espásticos, que exigem procurados, poder-se-ia supor que nos achávamos diante de um caso de atrofia muscular do tipo AranDuchenne. Mas, atendendo que a paresia não guardava relação com a atrofia e, sobretudo, 
tendo em conta a vivacidade dos reflexos, fiz o diagnóstico de Moléstia de Charcot, de tipo amyotrophico".

A partir da descrição do Dr. Cypriano Freitas, vários casos foram registrados na literatura médica nacional que contribuíram para o conhecimento da manifestação da ELA em nosso meio.

Em julho de 1913, Eduardo Monteiro, apresentou "um caso de esclerose lateral amyotrophica" na Gazeta Clínica de São Paulo onde registra que o início da afecção se deu no membro superior direito.

O Dr. Aloysio de Castro, professor de clínica médica da Universidade do Rio de Janeiro. Publicou, em 1914, o "Tractado de Semiótica Nervosa" que apresenta a descrição da Esclerose Lateral Amyotróphica (Mal de Charcot).

Em 1915, o Dr. Gonçalves Vianna escreveu suas observações “A respeito de um caso de Mal de Charcot (Typo Mixto)", na Revista dos Cursos da Faculdade de Medicina de Porto Alegre.

O primeiro caso de ELA bulbar foi apresentado, em 1916, pelo Dr. Raul Moreira da Silva em sua tese, aprovada com distinção, intitulada "Mal de Charcot (A Respeito de Um Caso de Início bulbar)" apresentada a Faculdade de Medicina de Porto Alegre/RS. Tratava-se de Paciente do sexo masculino, 56 anos de idade, branco, europeu natural de Duppau (Boemia), residente na cidade de Porto Alegre/RS, exercia a profissão de Farmacêutico. Os sintomas iniciaram com o sentir certa dificuldade em deglutir os alimentos, em novembro de 1914.

Os Doutores Luiz Guedes e Ney Cabral apresentaram, em 1918, um “Caso Clínico de Aspecto de E.L.A" em Porto Alegre/RS. Revista dos Cursos da Faculdade de Medicina de Porto Alegre.

Ainda do Rio Grande do Sul, em 1919, o Dr. Odino Lelio Pinós Duarte apresentou sua tese sobre a dissertação de "Em torno de um caso de Esclerose Lateral Amyotrophica (forma paraplegica)" à Faculdade de Medicina de Porto Alegre/RS.

Em 1924, os Doutores C. Trétiakoff e Moacir F. Amorim publicaram em francês no jornal Memórias do Hospicio Juquery - São Paulo, "Um cas de sclérose latérale amyotrophique pseudo-polynevritique, observée chez une alienée, atteinte de turbérculose intestinale" (Um caso de esclerose lateral amiotrófica pseudo-polineuritica, observado em uma 
alienada, portadora de tuberculose intestinal), em que apresentam os estudos de "autopsia e exame histopatológico" do Sistema Nervoso Central.

O Dr. Gonçalves Vianna, professor catedrático de anatomia e fisiologia patológica da Faculdade de Medicina de Porto Alegre, publicou no livro Clínica Neurológica, em 1925, "Em tôrno de dois casos de esclerose lateral amyotrophica (Molestia de Charcot)". Trata-se de dois estudos realizados na Faculdade de Medicina de Porto Alegre, em 1915 e 1916, o primeiro publicado na revista da faculdade e o segundo estudo realizado com alunos do quinto ano de medicina, que resultou na tese de Raul Moreira da Silva.

Em 1926, o Dr. Carlos da Motta Rezende apresenta sua tese de livre docência a Faculdade de Medicina do Rio de Janeiro, intitulada "Contribuições ao estudo da esclerose lateral amyotrophica (Doença de Charcot)".

No mês de outubro de 1927, o jornal Brazil-Médico publica o artigo dos Doutores F. Espozel e Teixeira Mendes - "Sobre um caso de esclerose lateral amyotrophica de início pseudo-polyneurítico" ocorrido na cidade do Rio de Janeiro. Os autores chamam a atenção para "a errônea noção de que a doença de Charcot é rara entre nós".

Também em 1927, o Dr. Paulino W. Longo apresenta sua tese à Faculdade de Medicina de São Paulo, sob o título de "Contribuições ao estudo da esclerose lateral amyotrophica", em que registra oito casos, e discute a natureza infecciosa da ELA. Pela importância do estudo, a Sociedade de Medicina e Cirurgia conferiu-lhe a medalha de ouro "Dr. Sergio Meira".

Dois trabalhos foram publicados em 1929: O primeiro em fevereiro no Arquivo Brasileiro de Medicina, "Doença de Charcot" dos Doutores Waldomiro Pires e Aloizio Marques. O segundo em agosto no Jornal Brasil-Medico, o relatório que foi apresentado em, junho do mesmo ano, no Congresso de Neurologia, Psiquiatria e Medicina Legal, no centenário da Academia Nacional de Medicina, dos conceituados professores da Faculdade de Medicina do Rio de Janeiro, Antonio Austregesilo e F. Esposel - "Contribuições Brasileiras ao Estudo de Esclerose Lateral Amyotrophica" em que tecem comentários sobre a "etiologia, pathogenia e physiologia pathologica", sobre os trabalhos publicados no Brasil e apresentam quatro casos clínicos. Afirmam em suas conclusões: “1. A opinião de raridade da esclerose amyotrophica no Brasil desapareceu com o apuro diagnóstico e a multiplicidade e publicações a respeito. 2. Apezar dos autores clássicos dizerem que não há alterações da sensibilidade na E.L.A., as contribuições brasileiras e americanas referem-nas, sobretudo na fórma pseudo-polyneuritica. 
3. A anatomia pathologica revela varias lesões que ultrapassam os systemas motores, o que explica o facto acima referido. 4. A observação brasileira não apura a hereditariedade, o elemento familiar, nem até agora determinou o agente causal da doença."

Em 1930, o Prof. Dr. Antonio de Moraes Austregesilo (Austragesilo Filho), apresenta sua tese de livre docência a congregação da Faculdade de Medicina do Rio de Janeiro, intitulada "Alterações da sensibilidade na doença de Charcot" onde apresenta seis casos clínicos, desses, quatro casos já publicados, em 1929.

No ano de 1935, é publicada a segunda edição do livro Semiótica Nervosa do Professor Aloysio de Castro. A ELA é apresentada de forma ilustrada no capítulo Semiotica das Atrofias Musculares - Amyotrophias Deuteropathias.

Tabela 1 - Contribuições brasileiras ao estudo da ELA - número de casos por Estado de 1909 a 1935.

\begin{tabular}{cccc}
\hline & Rio de Janeiro & São Paulo & Rio Grande do Sul \\
\hline Ano & casos & casos & casos \\
1909 & 01 & 01 & \\
1913 & & & 01 \\
1915 & & & 01 \\
1916 & & 01 & 01 \\
1918 & & & 01 \\
1919 & 01 & 08 & 02 \\
1924 & 04 & & \\
1925 & 04 & & \\
1927 & & 10 & 06 \\
1929 & 10 & & \\
1930 & &
\end{tabular}

2 - Estudos Epidemiológicos

Os estudos epidemiológicos aparecem na literatura médica nacional somente a partir da década de 1980. Até o momento atual foram realizados seis estudos epidemiológicos regionais e um estudo nacional, conforme apresentamos a seguir.

a) Estudos Epidemiológicos Regionais:

1 - O primeiro estudo - Aspectos epidemiológicos da esclerose lateral amiotrófica na cidade do Rio de Janeiro, foi realizado em 1983, pelo Dr. José Mauro Braz Lima e colaboradores. O estudo foi baseado nos dados de morbidade e mortalidade do Instituto de Neurologia Deolindo Couto (INDC/UFRJ), correspondentes a um período de 15 anos (1963 a 1977) de 136 pacientes com ELA. 92 homens (67.6\%) e 44 mulheres (32.2\%) uma taxa de 2:1. 
A média de Idade foi de 48,38 anos, sendo que 51,14\% tinham idade menor que 50 anos. A maioria eram brancos $(63,97 \%)$, seguidos de $20,58 \%$ de pardos e $15,44 \%$ de negros.

O Coeficiente de Mortalidade 1965 - 1974 (10 anos), variou de 0,3 - 0,9/100,000.

Os sintomas iniciais começaram nos membros superiores em 50,73\% dos pacientes, nos membros inferiores em $34,55 \%$ e, disartia e disfagia em $14,70 \%$. As formas clínicas do início da doença são mostradas na tabela 2 .

Tabela 2 - Formas clínicas de 136 pacientes com ELA no Instituto Deolindo Couto no período de 1963 a 1977.

\begin{tabular}{lccc}
\hline Formas Clínicas & No & Homens (\%) & Mulheres (\%) \\
\hline Espinhal & 106 & $75(55,14)$ & $31(22.79)$ \\
Bulbar & 20 & $11(8,08)$ & $9(6.61)$ \\
Pseudo-Polineuritica & 10 & $6(4,41)$ & $4(2.94)$ \\
TOTAL & $\mathbf{1 3 6}$ & $\mathbf{9 2}$ & $\mathbf{4 4}$ \\
\hline
\end{tabular}

Os demais estudos são mencionados pela ordem do ano de publicação, são eles:

2 - Realizado no Rio de Janeiro - RJ em 1991, abrangendo um período de 7 anos (1979 - 1986), por Gomes M. M. - Envelhecimento e o aumento das doenças amiotróficas: Epidemiologia das doenças (crônicas) das células do corno anterior da medula.

3 - Realizado em São Paulo - SP em 1998, abrangendo um período de 6 anos (1991 1997), por Moraes L, Goldbaum M, Silva HCA, Callegaro D. - Taxa de incidência de esclerose lateral amiotrófica na cidade de São Paulo.

4 - Realizado em Fortaleza - CE em 1999, abrangendo um período de 9 anos (1980 1999), por Castro-Costa CM, Oriá RB, Machado-Filho JA et al. - ELA. Análise clínica de 78 casos de Fortaleza (nordeste do Brasil).

5 - Realizado em Salvador - BA em 2000, abragendo um período de 2 anos (1997 1999), por Antônio de Souza Andrade e colaboradores - Perfil clínico do paciente com esclerose lateral amiotrófica na Bahia - Brasil.

6 - Realizado em Curitiba - PR em 2002, abrangendo um período de 14 anos (1977 2001), por Lineu César Werneck e colaboradores - Estudo clínico-epidemiológico de 226 casos de ELA. 
b) Estudo Epidemiológico Nacional:

O único estudo epidemiológico nacional foi realizado em 1998 por iniciativa da ABRELA e Aventis Farma, com a participação dos principais centros de referência e de 168 neurologistas de várias regiões do país.

Os autores deste trabalho foram: Flávia Dietrich-Neto, Dagoberto Callegaro, Elza Dias Tosta, Helga Almeida Silva, Maria Elizabeth Ferraz, José Mauro Braz Lima, Acary Souza Bulle Oliveira.

O tema: Esclerose Lateral Amiotrófica no Brasil: registro nacional, 1998.

Foram incluídos no trabalho 443 pacientes de várias regiões do Brasil, sendo 259 homens $(58,5 \%)$ e mulheres 184(41,5\%), com uma taxa de 1.4/1. A média de idade foi 52 anos \pm 13 , e a mediana 52 anos de idade. A forma clínica inicial mais freqüente encontrada no estudo foi a espinhal. Apresentamos a tabela $3 \mathrm{com}$ as formas clínicas e as demais características da maneira como aparece no artigo.

Os autores concluem que, as características epidemiológicas da doença são semelhantes a dos estudos internacionais, exceto para a idade dos primeiros sintomas (pacientes brasileiros são mais jovens).

Tabela 3 - Distribuição do sexo de acordo com as Formas Clínicas, tempo do primeiro sintoma até o diagnóstico, idade de início e sobreviventes.

\begin{tabular}{|c|c|c|c|}
\hline Características & Homens & Mulheres & Num. Total \\
\hline Forma Clínica* & $\mathrm{N}(\%)$ & $\mathrm{N}(\%)$ & $\mathrm{N}(\%)$ \\
\hline Bulbar & $38(15)^{* *}$ & $44(24) * *$ & $82(18.5)$ \\
\hline Apendicular & $187(73)$ & $118(65)$ & $306(69.1)$ \\
\hline Generalizada & $32(12)$ & $20(11)$ & $52(11.7)$ \\
\hline \multicolumn{4}{|c|}{$\begin{array}{l}\text { Tempo do primeiro sintoma até o } \\
\text { diagnóstico (meses): }\end{array}$} \\
\hline Média $( \pm$ SD) & $17(30)$ & $20(25)$ & $18(28)$ \\
\hline Mediana & 10 & 12 & 11 \\
\hline Máximo e mínimo & 0 a 332 & 0 a 210 & 0 a 332 \\
\hline \multicolumn{4}{|l|}{ Idade de início (anos) } \\
\hline Média ( \pm SD) & $50(13)$ & $54(13)$ & $52(13)$ \\
\hline Mediana & 51 & 54 & 52 \\
\hline \multicolumn{4}{|l|}{ Sobreviventes } \\
\hline $\begin{array}{l}\text { (23 pacientes) } \\
\text { (meses) }\end{array}$ & $\mathrm{N}=13$ & $\mathrm{~N}=10$ & \\
\hline Média $( \pm S D)$ & $49(46)$ & $33(18)$ & $42(37)$ \\
\hline Máximo e mínimo & 7 a 60 & 9 a 191 & 7 a 191 \\
\hline
\end{tabular}


3 - Atuação da Associação Brasileira de Esclerose Lateral Amiotrófica - ABRELA

Em 1998, Acary Souza Bulle Oliveira, neurologista e responsável pelo Setor de Investigação de Doenças Neuromusculares da Universidade Federal de São Paulo - Escola Paulista de Medicina (UNIFESP-EPM), idealizou implantar no Brasil um modelo de suporte ao paciente com ELA/DNM, já existente nos Estados Unidos. Essa idéia mobilizou um grupo de neurologistas que reunidos deram o primeiro passo para a criação da ABRELA: iniciativa pioneira de mobilizar a sociedade civil, científica e política da importância de conhecer a doença, sua manifestação na população brasileira e a necessidade de usar todos os recursos disponíveis de efeito comprovado para o tratamento. A proposta era que a ABRELA tivesse uma atuação em todo o território nacional de informação e orientação.

Ainda em 1998, a ABRELA, participou do primeiro estudo epidemiológico brasileiro, visando fazer conhecidas às características da doença no nosso país e desde então tem participado de todos os eventos que envolvem a esclerose lateral amiotrófica.

Em 2001, a ABRELA participou, pela primeira vez, do Simpósio Internacional de ELA/DNM e, desde então, tem apresentado para a comunidade científica internacional vários estudos realizados no Brasil, e tem publicado por meio da parceria com a Sanofi-Aventis o resumo do simpósio internacional para divulgação no Brasil.

O primeiro Simpósio Brasileiro de ELA/DNM foi realizado pela ABRELA em 2002, com a participação dos Doutores José Mauro de Braz Lima, Acary de Souza Bulle, Mamede de Carvalho, Helga Cristina Almeida da Silva e Wilsom Marques Júnior. A ABRELA realiza simpósios, anualmente, visando mobilizar a comunidade científica e profissionais da saúde.

Hoje a ABRELA é membro da Aliança Internacional das Associações de ELA/DNM e da Associação Internacional de ELA/DNM, e está em fase de expansão de suas atividades, com a criação das ARELAS (Associação Regional de ELA) - ARELA/RS (Associação Regional de ELA do Estado do Rio Grande do Sul) já implantada e ARELA/MG (Associação Regional de ELA do Estado de Minas gerais) em implantação, o objetivo é beneficiar um número maior de pacientes.

\section{Referências Bibliográficas}

1.Freitas CS. Sobre um caso de esclerose lateral amyotrophica. Brazil-Medico, anno XXIII, num 42, pag. 421-22, 8 de novembro de 1909. Publicado também no Arch Bras Psychiatr Neurol Med Legal 1910: 71. 
2.Monteiro E. Um caso de esclerose lateral amyotrophica. Gazeta Clínica de São Paulo, 1913, p.144.

3.Castro A. Tractado de Semiótica Nervosa: Semiótica das formas exteriores e das desordens motoras. Rio de Janeiro: F. Briguiet \& Cia., Livreiros -Editores, 1914.

4.Vianna G. A respeito de um caso de Mal de Charcot (Typo Mixto). Revista dos Cursos da Faculdade de Medicina de Porto Alegre, 1915.

5.Silva RM. Mal de Charcot (A Respeito de Um Caso de Início bulbar)" - These apresentada a Faculdade de Medicina de Porto Alegre/RS, em 2 de outubro de 1916.

6.Guedes L, Cabral N. Caso Clínico de Aspecto de E.L.A.”. Revista dos Cursos da Faculdade de Medicina de Porto Alegre, 1918.

7.Duarte OLP. Em torno de um caso de Esclerose Lateral Amyotophica (forma paraplegica)" These apresentada a Faculdade de Medicina de Porto Alegre/RS em Dezembro de 1919.

8.Trétiakoff C, Amorim MF. Um cas de sclérose latérale amyotrophique pseudo-polynevritique, observée chez une alienée, atteinte de turbérculose intestinale. Memórias do Hospício de Juquery, pp.259-266, São Paulo, 1924.

9.Vianna G. Em tôrno de dois casos de esclerose lateral amyotrophica (Molestia de Charcot). Clínica Neurológica, Porto Alegre: Editores Barcellos, Bertaso \& CIA, 1a. Ed., 1925, pp 219-236.

10.Rezende CM. Contribuições ao estudo da esclerose lateral amyotrophica (Doença de Charcot) These de docencia livre apresentada a Faculdade de Medicina do Rio de Janeiro, 1926.

11.Espozel F, Mendes T. Sobre um caso de esclerose lateral amyotrophica de início pseudopolyneurítico. Brazil -Medico, anno XLI, num 44, pp. 1147-1149, outubro de 1927.

12.Longo PW. Contribuições ao estudo da esclerose lateral amyotrophica. These apresentada a Faculdade de Medicina de São Paulo,1927.

13.Pires WP, Marques A. Doença de Charcot. Arch Bras Med 1929:74-81.

14.Austregesilo A, Esposel F. Contribuições Brasileiras ao Estudo de esclerose Lateral Amyotrophica. Brasil-Medico anno XLII n.34, 1929, p.999-1006.

15.Austregesilo AM. (Austregésilo Filho). Alterações da sensibilidade na doença de Charcot. These de livre docencia apresentada a congregação da Faculdade de Medicina do Rio de Janeiro, 1930.

16.Castro A - SEMIOTICA NERVOSA. Semiótica das formas exteriores e das perturbações motoras. 2a. ed. revista e augmentada. Rio de Janeiro: F. Briguiet \& Cia, 1935.

17.Lima JMB, Mesquita N, Duro LAA, Furtado AB. Epidemiological aspects of amyotrophic lateral sclerosis in Rio de Janeiro city. Rev bras Neurol 1983; 19(3):75-78.

18.Gomes MM. Envelhecimento e o aumento das doenças amiotróficas: epidemiologia das doenças (crônicas) das células do corno anterior da medula. Arq Bras Med 1991;65:589-594. 
19.Moraes L, Goldbaum M, Silva HCA, Callegaro D. Incidence rate of amyotrophic lateral sclerosis (ALS) in São Paulo city, Brazil, 1991 - 1997. Arq Neuropsiquiatr 1998; 58(Supl 1):343.

20.Castro-Costa CM, Oriá RB, Machado-Filho JA. Amyotrophic lateral sclerosis. Clinical analysis of 78 cases from Fortaleza (northeastern Brazil). Arq Neuropsiquiatr 1999; 57:761-774.

21.Andrade Filho AS, Lima JMB, Matos AES. Perfil clínico do paciente com Esclerose Lateral Amiotrófica na Bahia - BRASIL. Rev Bras Neurol Psiquiatr 2000; 4(3):81-85.

22.Werneck LC, Bezerra R, Silveira Neto O, Scola RH. Esclerose Lateral Amiotrófica. Estudo Clínicoepidemiológico de 226 casos. O Dendrito 2002; 8:7.

23.Dietrich-Neto F, Callegaro D, Tosta ED, Silva HCA, Balallai MEF, Lima JMB, et al. Amyotrophic Lateral Sclerosis in Brazil: 1998 National Survey. Arq Neuro-psiquiatr 2000; 58:607-615. 